\begin{tabular}{|c|l|}
\hline Title & Domain dependent monotonicity formula for a singular perturbation problem \\
\hline Author(s) & Tonegawa, Y. \\
\hline Citation & Hokkaido University Preprint Series in Mathematics, 554, 1-14 \\
\hline Issue Date & 2002-07 \\
\hline DOI & 10.14943/83699 \\
\hline Doc URL & http:/hdl.handle.net/2115/69303 \\
\hline Type & bulletin (article) \\
\hline File Information & pre554.pdf \\
\hline
\end{tabular}

Instructions for use 
DOMAIN DEPENDENT MONOTONICITY FORMULA FOR A SINGULAR

PERTURBATION PROBLEM

Yoshihiro Tonegawa

Series \#554. July 2002 


\section{HOKKAIDO UNIVERSITY PREPRINT SERIES IN MATHEMATICS}

\#528 T. Nakazi and T. Yamamoto, Two dimensional commutative Banach algebras and von Neumann inequality, 18 pages. 2001.

\#529 Y. Giga, N. Ishimura and Y. Kohsaka, Spiral solutions for a weakly anisotropic curvature flow equation, 16 pages. 2001.

\#530 Y. Giga and P. Rybka, Quasi-static evolution of 3-D crystals grown from supersaturated vapor, 16 pages. 2001.

\#531 Y. Tonegawa, Remarks on convergence of the Allen-Cahn equation, 18 pages. 2001.

\#532 T. Suwa, Characteristic classes of singular varieties, 26 pages. 2001.

\#533 J. Escher, Y. Giga and K. Ito, On a limiting motion and self-intersections for the intermediate surface diffusion flow, 20 pages. 2001 .

\#534 Y.-H. R. Tsai, Y. Giga and S. Osher, A level set approach for computing discontinuous solutions of a class of Hamilton-Jacobi equations, 30 pages. 2001.

\#535 A. Yamagami, On Gouvêa's conjecture in the unobstructed case, 19 pages. 2001.

\#536 A. Inoue, What does the partial autocorrelation function look like for large lags, 27 pages. 2001.

\#537 T. Nakazi and T. Yamamoto, Norm of a linear combination of two operators of a Hilbert space, 16 pages. 2001.

\#538 Y. Giga, On the two-dimensional nonstationary vorticity equations, 12 pages. 2001.

\#539 M. Jinzenji, Gauss-Manin system and the virtual structure constants, 25 pages. 2001.

\#540 H. Ishii and T.Mikami, Motion of a graph by $R$-curvature, 28 pages. 2001.

\#541 M. Jinzenji and $\mathrm{T}$. Sasaki, $\mathcal{N}=4$ supersymmetric Yang-Mills theory on orbifold- $T^{4} / \mathbf{Z}_{2}$ : higher rank case, 17 pages. 2001.

\#542 T. Nakazi, The Nevanlinna counting functions for Rudin's orthogonal functions, 7 pages. 2001.

\#543 K. Sugano, On H-separable extensions of QF-3 rings, 7pages. 2001.

\#544 A. Arai, Non-relativistic limit of a Dirac-Maxwell operator in relativistic quantum electrodynamics, 27 pages. 2001.

\#545 O. Sawada, On time-local solvability of the Navier-Stokes equations in Besov spaces, 30 pages. 2001.

\#546 C. M. Elliott, Y. Giga, and S. Goto, Dynamic boundary conditions for Hamilton-Jacobi equations, 27 pages. 2001.

\#547 Y. Nakano, Minimizing coherent risk measures of shortfall in discrete-time models with cone constraints, 22 pages. 2002.

\#548 K. tachizawa, A generalization of the Lieb-Thirring inequalities in low dimensions, 13 pages. 2002.

\#549 T. Nakazi, Absolute values and real parts for functions in the Smirnov class, 8 pages. 2002.

\#550 T. Nakazi and T. Watanabe, Properties of a Rubin's orthogonal function which is a linear combination of two inner functions, 9 pages. 2002.

\#551 T. Ohtsuka, A level set method for spiral crystal growth, 24 pages. 2002.

\#552 M.-H. Giga and Y. Giga, Minimal vertical singular diffusion preventing overturning for the Burgers equation, 18 pages. 2002 .

\#553 Y. Giga and P. Rybka, Berg's effect, 12 pages. 2002. 


\title{
DOMAIN DEPENDENT MONOTONICITY FORMULA FOR A SINGULAR PERTURBATION PROBLEM
}

\author{
YOSHIHIRO TONEGAWA
}

\begin{abstract}
We consider a singular perturbation problem arising in the scalar phase field model with Neumann boundary conditions on convex domains, and establish a monotonicity formula for general critical points. This gives the Hausdorff distance convergence of the phase boundaries as the parameters tend to zero. We apply the result to stable critical points defined on strictly convex domains, showing that the limit interfaces for stable critical points are necessarily connected.
\end{abstract}

\section{INTRODUCTION}

In this paper, we consider the variational problem associated with the energy

$$
E_{\varepsilon}(u)=\int_{\Omega} \frac{\varepsilon}{2}|\nabla u|^{2}+\frac{W(u)}{\varepsilon},
$$

where $\Omega \subset \mathbb{R}^{n}$ is a bounded domain, $u: \Omega \rightarrow \mathbb{R}, W: \mathbb{R} \rightarrow \mathbb{R}^{+} \cup\{0\}$ is a non-convex potential function and $\varepsilon>0$ is a small parameter. This is a typical energy modeling the phase separation phenomena within the van der Waals - Cahn - Hilliard theory ([1]). In this context, $u$ represents the density of a multi-phase fluid, where the zero points of $W$ correspond to stable fluid phases, and the free-energy $E_{\varepsilon}(\cdot)$ depends both on the density potential and the density gradient. The constraint to be imposed in this paper is the integral constraint, which is equivalent to fixing the total mass of the fluid in $\Omega$. Let

$$
A_{m}=\left\{u \in H^{1}(\Omega) \mid \int_{\Omega} u=m\right\},
$$

where $m \in \mathbb{R}$ is given and $H^{1}(\Omega)=\left\{u \in L^{2}(\Omega) \mid \nabla u \in L^{2}(\Omega)\right\}$. Consider the minimization problem, for $\varepsilon>0$ small,

$$
\inf _{v \in A_{m}} E_{\varepsilon}(v)
$$

Partly supported by Grant-in-Aid for Young Scientists (A) grant no. 14702001. 
The standard direct method of minimization shows the existence of a minimizer $u_{\varepsilon}$, satisfying

$$
\begin{cases}-\varepsilon \Delta u_{\varepsilon}+\frac{W^{\prime}\left(u_{\varepsilon}\right)}{\varepsilon}=\lambda_{\varepsilon} & \text { on } \Omega, \\ \frac{\partial u_{s}}{\partial \nu}=0 & \text { on } \partial \Omega,\end{cases}
$$

where $\lambda_{\varepsilon} \in \mathbb{R}$ is the Lagrange multiplier. The energy $E_{\varepsilon}\left(u_{\varepsilon}\right)$ can be bounded uniformly in $0<\varepsilon<1$, since one can construct a family of suitable comparison functions with finite energy. One expects that $u_{\varepsilon}$ has a transition region of $\mathcal{O}(\varepsilon)$-thickness, and as $\varepsilon \rightarrow 0$, it should approach to some hypersurface with least possible area with the given constraint. The rigorous study of the asymptotic behavior of $u_{\varepsilon}$ was first done by Modica [7], Sternberg [10] and others via the technique of $\Gamma$-convergence. These authors show in general that there exists a limit $u_{0}$ which takes discrete values a.e. $L^{n}$ on $\Omega$, and the interface minimizes the hypersurface area with the integral constraint thus it is in fact a constant mean curvature hypersurface. With a suitable growth condition on $W$, the convergence of $u_{\varepsilon}$ to the limit $u_{0}$ as $\varepsilon \rightarrow 0$ is in $L^{p}(\Omega)$ for any $1 \leq p<\infty$. On the other hand, the convergence of the interface region to the limit constant mean curvature hypersurface is not clear through this approach. With the assumption that $\Omega$ is convex, we improve on these results by showing a suitable energy monotonicity formula well-known in an analogous context for minimal surface theory, and show that the interface region for $u_{\varepsilon}$ converge to the limit interface in the Hausdorff distance topology, up to the boundary. As an application, we strengthen the result of Sternberg \& Zumbrun [11] on the structure of stable equilibriums, showing that the limit interface of stable critical points of $E_{\varepsilon}(\cdot)$ is necessarily connected if $\Omega$ is strictly convex. This shows that any interface with more than one component cannot be the limit interface of those of stable critical points. We show the monotonicity formula itself holds for any critical points for $E_{\varepsilon}(\cdot)$, including unstable critical points, and the shape of $W$ is rather unimportant as well.

\section{ASSUMPTION AND STATEMENTS OF RESUltS}

We assume that

(A.1) $\partial \Omega$ is $C^{3, \alpha}$-manifold for some $0<\alpha<1$ and $\Omega$ is convex.

(A.2) Let $\left\{\varepsilon_{i}\right\}_{i=1}^{\infty} \subset \mathbb{R}^{+}$be positive numbers such that $\lim _{i \rightarrow \infty} \varepsilon_{i}=0$ and $u^{\varepsilon_{i}} \in C^{3}(\bar{\Omega})$ satisfies

$$
\begin{cases}-\varepsilon_{i} \Delta u^{\varepsilon_{i}}+\frac{W^{\prime}\left(u^{\varepsilon_{i}}\right)}{\varepsilon_{i}}=\lambda_{i} & \text { on } \Omega, \\ \frac{\partial u^{\varepsilon_{i}}}{\partial \nu}=0 & \text { on } \partial \Omega .\end{cases}
$$


(A.3) There exist constants $E_{0}, c_{1}$ and $c_{2}$ such that

$$
E_{\varepsilon_{i}}\left(u^{\varepsilon_{i}}\right) \leq E_{0},\left.|| u^{\varepsilon_{i}}\right|_{L^{\infty}} \leq c_{1},\left|\lambda_{i}\right| \leq c_{2}
$$

for all $i$.

(A.4) $W \in C^{3}(\mathbb{R}), W \geq 0$ and there are $a_{1}<\cdots<a_{N}$ and $\kappa>0$, such that $W\left(a_{j}\right)=0$ and $W^{\prime \prime}\left(a_{j}\right) \geq \kappa$ for $j=1, \cdots, N$ and $W>0$ on $\mathbb{R} \backslash\left\{a_{1}, \cdots, a_{N}\right\}$.

Note that we do not assume energy minimality of $u^{\varepsilon_{i}}$ here.

Remark 1. Assume that there exist constants $2<k<\frac{2 n}{n-2}$ and $c>0$ such that $c|x|^{k} \leq W(x) \leq c^{-1}|x|^{k}$ and $c|x|^{k-1} \leq\left|W^{\prime}(x)\right| \leq c^{-1}|x|^{k-1}$ for all large $|x|$ and that $E_{0}$ and $m$ are given. For any sequence of critical points $\left\{u^{\varepsilon_{i}}\right\}_{i=1}^{\infty} \subset H^{1}(\Omega)$ with $E_{\varepsilon_{i}}\left(u^{\varepsilon_{i}}\right) \leq E_{0}$ and $\int_{\Omega} u^{\varepsilon_{i}}=m$, one can show that there exist $c_{1}$ and $c_{2}$ satisfying (A.2) and (A.3) (see $[5$, Section 6.1]).

Next, since $\lim \sup _{i \rightarrow \infty} \int_{\Omega}\left(\frac{\varepsilon_{i}\left|\nabla u^{\varepsilon_{i}}\right|^{2}}{2}+\frac{W\left(u^{\varepsilon_{i}}\right)}{\varepsilon_{i}}\right) \leq E_{0}$, there exists a subsequence (denoted again by $\left\{u^{\varepsilon_{i}}\right\}_{i=1}^{\infty}$ ) and a Radon measure $\mu$ such that, for any $\phi \in C_{c}\left(\mathbb{R}^{n}\right)$,

$$
\lim _{i \rightarrow \infty} \int_{\Omega}\left(\frac{\varepsilon_{i}\left|\nabla u^{\varepsilon_{i}}\right|^{2}}{2}+\frac{W\left(u^{\varepsilon_{i}}\right)}{\varepsilon_{i}}\right) \phi=\int_{\bar{\Omega}} \phi d \mu .
$$

Let spt $\mu$ be the support of $\mu$ as a measure on $\mathbb{R}^{n}$ and not only on $\Omega$. We denote $B_{r}(x)=\{y \in \bar{\Omega}|| y-x \mid<r\}$. For this $\mu$, we show

Theorem 1. There exist $0<D_{1} \leq D_{2}<\infty$ depending only on $W, \Omega$, $E_{0}, c_{1}$ and $c_{2}$ such that

$$
D_{1} \leq \frac{1}{\omega_{n-1} r^{n-1}} \mu\left(B_{r}(x)\right) \leq D_{2}
$$

for all $x \in \operatorname{spt} \mu$ and $0<r<$ diameter $\Omega$. Here, $\omega_{n-1}$ is the $(n-1)$ dimensional measure of the unit ball in $\mathbb{R}^{n-1}$.

By the standard result in measure theory $([9])$, there exists an $\mathcal{H}^{n-1}$ measurable function $\theta(x)$ such that $\mu=\theta(x) \mathcal{H}^{n-1}$, with $D_{1} \leq \theta(x) \leq$ $D_{2}, \mathcal{H}^{n-1}$ a.e. on spt $\mu$ and $\theta(x)=0$ on $\bar{\Omega} \backslash$ spt $\mu$. It also follows that $\mathcal{H}^{n-1}(\operatorname{spt} \mu)<\infty$.

As for the convergence of the interfaces, we have the following;

Theorem 2. For any $\delta_{0}>\delta>0,\left\{x \in \bar{\Omega} \mid W\left(u^{\varepsilon_{i}}\right) \geq \delta\right\}$ converge to spt $\mu$ in the Hausdorff distance topology. Here, we fix $\delta_{0}>0$ to be such that $W(s) \leq \delta_{0}$ implies $W^{\prime \prime}(s) \geq \kappa / 2$. 
Recall that sets $\left\{K_{i}\right\}_{i=1}^{\infty}$ converge to $K$ in the Hausdorff distance topology if for any $\gamma>0$, the $\gamma$-neighborhood of $K$ includes $K_{i}$ and $\gamma$ neighborhood of $K_{i}$ includes $K$ for all sufficiently large $i$. Among other things, this gives a uniform convergence of $u^{\varepsilon_{i}}$ away from the support of $\mu$ :

Theorem 3. For any $\gamma>0$, let $(\text { spt } \mu)_{\gamma} \subset \mathbb{R}^{n}$ be the $\gamma$-neighborhood of spt $\mu$ in $\mathbb{R}^{n}$. For each connected component of $\Omega \backslash \overline{(\text { spt } \mu)_{\gamma}}, u^{0}=$ $\lim _{i \rightarrow \infty} u^{\varepsilon_{i}}$ is constant and equal to one of $a_{1}, \cdots, a_{N} .\left\{u^{\varepsilon_{i}}\right\}_{i=1}^{\infty}$ converge to $u^{0}$ uniformly on $\Omega \backslash \overline{(s p t \mu)_{\gamma}}$.

Assume further that $W$ is double-well shaped, i.e., $N=2$ and $W^{\prime}$ has only three zeros, and that $\left\{u^{\varepsilon_{i}}\right\}_{i=1}^{\infty}$ are stable critical points, i.e.,

$$
\left.\frac{d^{2}}{d t^{2}}\right|_{t=0} E_{\varepsilon_{i}}\left(u^{\varepsilon_{i}}+t v\right) \geq 0
$$

for all $v \in H^{1}(\Omega)$ with $\int_{\Omega} v=0$. Assume that $\Omega$ is strictly convex. Also assume that $m$ is fixed and $\frac{m}{L^{n}(\Omega)} \in\left(a_{1}, a_{2}\right)$. In this case, we may improve the results due to Sternberg \& Zumbrun in the following form:

Theorem 4. spt $\mu$ is connected in $\bar{\Omega}$.

This shows that any interface consisting of more than one component cannot be a limit interface of stable critical points with a volume constraint.

\section{Proof of global Monotonicity Formula AND the COROLLARIES}

Here, we establish an energy monotonicity formula for a properly scaled energy $E_{\varepsilon}(\cdot)$. In doing so, one important step is to show that the term $\frac{\varepsilon}{2}|\nabla u|^{2}-\frac{W(u)}{\varepsilon}$ is bounded from above in $L^{\infty}$-norm independent of $\varepsilon$. In case $\Omega$ is convex, one can apply the maximum principle argument to show that it is in fact bounded from above. To obtain the monotonicity formula up to the boundary, one needs to adopt a suitable reflection argument analogous to the method due to Grüter and Jost [3]. In the following, we drop the subscript $i$ from $\varepsilon_{i}, \varepsilon_{i}$ from $u^{\varepsilon_{i}}$ and etc.

We recall a result which has appeared, for example, in $[2,11]$.

Lemma 1. Let $B(\cdot, \cdot)$ be the second fundamental form of $\partial \Omega$. If $\frac{\partial u}{\partial \nu}=0$ on $\partial \Omega$, then $\frac{\partial}{\partial \nu}|\nabla u|^{2}=2 B(\nabla u, \nabla u)$. In particular, if $\Omega$ is convex, then, $\frac{\partial}{\partial \nu}|\nabla u|^{2} \leq 0$ on $\partial \Omega$. 
Proof. After a suitable translation and rotation, we may assume that $x=0$ and $\partial \Omega$ is locally a graph over $\mathbb{R}^{n-1}$,

$$
\partial \Omega \cap B_{r}(0) \subset\left\{\left(x_{1}, \cdots, x_{n-1}, f\left(x_{1}, \cdots, x_{n-1}\right)\right\},\right.
$$

with $0=f(0, \cdots, 0), \nabla f(0, \cdots, 0)=0, \frac{\partial^{2} f}{\partial x_{i} \partial x_{j}}(0, \cdots, 0)=\delta_{i j} \kappa_{j}$. Here, $\kappa_{j}$ 's are the principal curvatures of $\partial \Omega$ at $x=0$. The unit normal to $\partial \Omega$ is $\frac{1}{\sqrt{1+|\nabla f|^{2}}}\left(-f_{x_{1}}, \cdots,-f_{x_{n-1}}, 1\right)$, so the boundary condition means

$$
u_{x_{n}}-\sum_{i=1}^{n-1} u_{x_{i}} f_{x_{i}}=0
$$

where the variables are $\left(x_{1}, \cdots, x_{n-1}, f\left(x_{1}, \cdots, x_{n-1}\right)\right)$. By differentiating with respect to $x_{j}, j=1, \cdots, n-1$, and evaluating at the origin, we obtain

On the other hand,

$$
u_{x_{n} x_{j}}=u_{x_{j}} \kappa_{j} .
$$

$$
\frac{\partial}{\partial \nu}|\nabla u|^{2}=2 \sum_{i=1}^{n} u_{x_{j}} u_{x_{j} x_{n}}=2 \sum_{j=1}^{n-1} u_{x_{j}} u_{x_{j} x_{n}}
$$

at the origin, so using the above, we obtain $\frac{\partial}{\partial \nu}|\nabla u|^{2}=2 \sum_{j=1}^{n-1}\left(u_{x_{j}}\right)^{2} \kappa_{j}$. In terms of the second fundamental form, this proves the stated results.

Next we prove

Proposition 1. Let $\widetilde{W}(u)=W(u)-\varepsilon \lambda u+\varepsilon c_{1} c_{2}$. Then,

$$
\frac{\varepsilon|\nabla u|^{2}}{2} \leq \frac{\widetilde{W}(u)}{\varepsilon}
$$

on $\Omega$.

In case the domain is $\mathbb{R}^{n}$, Modica proved such inequality in [6]. Here we prove it in case $\Omega$ is convex.

Proof. We rescale the domain by $1 / \varepsilon$ so the equation becomes

$$
\begin{cases}\Delta u=\widetilde{W}^{\prime}(u) & \text { on } \frac{1}{\varepsilon} \Omega \\ \frac{\partial u}{\partial \nu}=0 & \text { on } \frac{1}{\varepsilon} \Omega .\end{cases}
$$

Let $\xi=\frac{|\nabla u|^{2}}{2}-\widetilde{W}(u)$. Compute

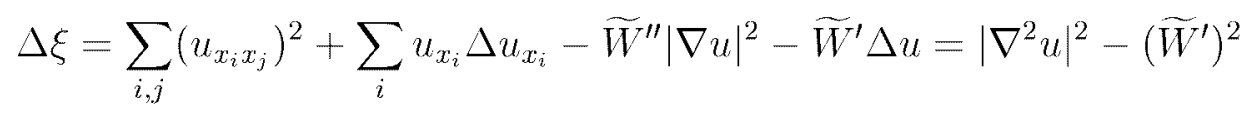

by the equation. On the other hand, by the Cauchy-Schwarz inequality,

$$
\sum_{j}\left(\sum_{i} u_{x_{i}} u_{x_{i} x_{j}}\right)^{2} \leq|\nabla u|^{2} \sum_{i, j}\left(u_{x_{i} x_{j}}\right)^{2}
$$


and by substituting $\xi_{x_{j}}=\sum_{i} u_{x_{i}} u_{x_{i} x_{j}}-\widetilde{W}^{\prime} u_{x_{j}}$,

$$
\sum_{j}\left(\xi_{x_{j}}+\widetilde{W}^{\prime} u_{x_{j}}\right)^{2} \leq|\nabla u|^{2} \sum_{i, j}\left(u_{x_{i} x_{j}}\right)^{2} .
$$

Hence, on $\{|\nabla u|>0\}$,

$$
\Delta \xi \geq|\nabla u|^{-2} \sum_{j}\left(\xi_{x_{j}}+\widetilde{W}^{\prime} u_{x_{j}}\right)^{2}-\left(\widetilde{W}^{\prime}\right)^{2}=\frac{|\nabla \xi|^{2}}{|\nabla u|^{2}}+\frac{2 \widetilde{W^{\prime}} \nabla u}{|\nabla u|^{2}} \cdot \nabla \xi,
$$

and

$$
\Delta \xi-\frac{2 \widetilde{W}^{\prime} \nabla u}{|\nabla u|^{2}} \cdot \nabla \xi \geq 0
$$

Assume for a contradiction that $\left\{\frac{|\nabla u|^{2}}{2}>\widetilde{W}\right\} \cap \Omega$ is not empty. Let $x_{0} \in \bar{\Omega}$ be the maximum point of $\frac{|\nabla u|^{2}}{2}-\widetilde{W}$. Since $\widetilde{W}$ is chosen to be non-negative, we must have $|\nabla u|^{2}(x)>0$ in the neighborhood of $x_{0}$. If $x_{0} \in \partial \Omega, \frac{\partial \xi}{\partial \nu}\left(x_{0}\right)=\frac{1}{2} \frac{\partial}{\partial \nu}|\nabla u|^{2}-\frac{\partial}{\partial \nu} \widetilde{W}(u) \leq 0$ by Lemma 1 and the boundary condition $\frac{\partial u}{\partial \nu}=0$. By the Hopf maximum principle [4, Lemma 3.4], this leads to a contradiction. Interior maximum is also excluded by the same reason. By rescaling back, we obtain the desired inequality.

Once we obtain the estimate (3.1), we may prove (cf. $[7,5]$ )

Theorem 5. (Interior monotonicity formula) For all $x \in \Omega$ and $0<$ $r<\operatorname{dist}(x, \partial \Omega)$, we have

$$
\frac{d}{d r}\left(\frac{1}{r^{n-1}} \int_{B_{r}(x)} \widetilde{\mathcal{E}}\right) \geq 0
$$

Here, $\widetilde{\mathcal{E}}=\frac{\varepsilon|\nabla u|^{2}}{2}+\frac{\widetilde{W}}{\varepsilon}$.

For the convenience of the reader, we supply the proof.

Proof. Multiply both sides of the equation (2.1) by $\nabla u \cdot g$, where $g=$ $\left(g^{1}, \cdots, g^{n}\right) \in C_{c}^{1}\left(\Omega ; \mathbb{R}^{n}\right)$. Then, after two integrations by parts, we obtain

$$
\int_{\Omega}\left(\widetilde{\mathcal{E}} \operatorname{div} g-\varepsilon \sum_{i, j} u_{y_{i}} u_{y_{j}} g_{y_{i}}^{j}\right)=0
$$

Let $x=0$ by a suitable translation and let $g^{j}(y)=y_{j} \rho(|y|)$, where $\rho(|y|)$ is a smooth approximation to the characteristic function for $B_{r}(0) \subset \Omega$. Writing $r=|y|$, the above becomes

$$
\int_{\Omega}\left(\tilde{\mathcal{E}}\left(r \rho^{\prime}+n \rho\right)-\varepsilon \frac{\rho^{\prime}}{r}(y \cdot \nabla u)^{2}-\varepsilon|\nabla u|^{2} \rho\right)=0 .
$$


By letting $\rho \rightarrow \chi_{B_{r}(0)}$ and rearranging terms, we obtain

$-(n-1) \int_{B_{r}(0)} \tilde{\mathcal{E}}+r \int_{\partial B_{r}(0)} \tilde{\mathcal{E}}=\int_{B_{r}(0)}\left(\frac{\widetilde{W}}{\varepsilon}-\frac{\varepsilon|\nabla u|^{2}}{2}\right)+\frac{\varepsilon}{r} \int_{\partial B_{r}(0)}(y \cdot \nabla u)^{2}$.

By Proposition 1, the right-hand side is nonnegative. By dividing the above by $r^{n}$, we obtain the desired inequality.

To obtain a monotonicity formula for the rescaled energy up to the boundary, we adopt a method in [3], where the balls to measure the energy are reflected across the boundary $\partial \Omega$.

For $x \in \partial \Omega$, let $\tau, \nu$ be subspaces in $\mathbb{R}^{n}$ defined by

$$
\tau(x)=\operatorname{Tan}_{x}(\partial \Omega), \quad \nu(x)=\left(\operatorname{Tan}_{x}(\partial \Omega)\right)^{\perp},
$$

where $\perp$ means the orthogonal complement. We identify $\tau$ and $\nu$ as the corresponding orthogonal projections from $\mathbb{R}^{n}, \tau=\left(\tau_{i j}\right), \nu=\left(\nu_{i j}\right)$. Let $\kappa_{\max }$ be the maximum absolute value of the principal curvatures of $\partial \Omega$. For $x \in \Omega \cap\left\{\operatorname{dist}(x, \partial \Omega)<\kappa_{\max }\right\}$, there exists a unique point $y \in \partial \Omega$ such that $|x-y|=\operatorname{dist}(x, \partial \Omega)$. Denote such point by $\zeta(x)$ and $s(x)=|x-\zeta(x)|$. One easily checks (see [4], for example)

Lemma 2. 1. $\zeta$ is $C^{2}$ on $\Omega \cap\left\{\operatorname{dist}(x, \partial \Omega)<\kappa_{\max }^{-1}\right\}$,

2. $\operatorname{For} \zeta(x)=\left(\zeta_{1}(x), \cdots, \zeta_{n}(x)\right)$,

$$
\left(\zeta_{i}(x)\right)_{x_{j}}=\tau_{i j}(\zeta(x))
$$

and

$$
\left|\left(\zeta_{i}(x)\right)_{x_{j} x_{k}}\right| \leq \kappa_{\max } s(x) /\left(1-\kappa_{\max } s(x)\right) .
$$

For $x \in \Omega \cap\left\{\operatorname{dist}\left(x, \partial \Omega<\kappa_{\max }^{-1}\right\}\right.$, we define the reflection of $x$ across $\partial \Omega$ to be

$$
\widetilde{x}=2 \zeta(x)-x .
$$

For $w \in \mathbb{R}^{n}$, define $\mathcal{J}_{x}: \mathbb{R}^{n} \rightarrow \mathbb{R}^{n}$ by

$$
\mathcal{J}_{x}(w)=\tau(\zeta(x)) \cdot w-\nu(\zeta(x)) \cdot w,
$$

which reflects the normal component of the vector $w$ across $\operatorname{Tan}_{\zeta(x)}(\partial \Omega)$. We also use the notation

$$
\widetilde{B}_{r}(a)=\{x \in \bar{\Omega}|| \widetilde{x}-a \mid<r\} .
$$

We prove

Theorem 6. (Boundary monotonicity formula) There exists $c_{3}$ which depends only on $n$ and $\Omega$ such that

$$
\frac{d}{d r}\left(\frac{e^{c_{3} r}}{r^{n-1}}\left(\int_{B_{r}(x)} \widetilde{\mathcal{E}}+\int_{\widetilde{B}_{r}(x)} \widetilde{\mathcal{E}}\right)\right) \geq 0
$$

for all $x \in \bar{\Omega} \cap\left\{\operatorname{dist}(x, \partial \Omega)<\left(3 \kappa_{\max }\right)^{-1}\right\}$ and $0<r<\left(3 \kappa_{\max }\right)^{-1}$. 
Proof. We multiply both sides of the equation (2.1) by $g \in C^{1}\left(\mathbb{R}^{n} ; \mathbb{R}^{n}\right)$, with $g \cdot \nu=0$ on $\partial \Omega$. Using the boundary condition $\frac{\partial u}{\partial \nu}=0$ and $g \cdot \nu=0$ on $\partial \Omega$ in the integration by parts, we again obtain

$$
\int_{\Omega}\left(\widetilde{\mathcal{E}} \operatorname{div} g-\varepsilon \sum_{i, j} u_{y_{i}} u_{y_{j}} g_{y_{i}}^{j}\right)=0
$$

Now we use $g$ defined by

$$
g^{i}(x)=\rho(r)(x-a)_{i}+\rho(\widetilde{r})\left(\mathcal{J}_{x}(\widetilde{x}-a)\right)_{i},
$$

where $r(x)=|x-a|, \widetilde{r}(x)=|\widetilde{x}-a|, \rho(r)$ is a function to be chosen. Since $r=\widetilde{r}$ and $-(x-a) \cdot \nu=\mathcal{J}_{x}(x-a) \cdot \nu$ on $\partial \Omega$, we have $g \cdot \nu=0$ on $\partial \Omega$. Since

$$
\begin{gathered}
g_{x_{j}}^{i}=\rho^{\prime}(r) \frac{(x-a)_{i}(x-a)_{j}}{r}+\rho(r) \delta_{i j}+\rho^{\prime}(\widetilde{r}) \frac{\mathcal{J}_{x}(\widetilde{x}-a)_{j} \mathcal{J}_{x}(\widetilde{x}-a)_{i}}{\widetilde{r}} \\
+\rho(\widetilde{r})\left\{\sum_{k}\left(\tau_{i k}-\nu_{i k}\right)_{x_{j}}(\widetilde{x}-a)_{k}+\delta_{i j}\right\}
\end{gathered}
$$

we obtain

$$
\begin{gathered}
\int_{\Omega}\left[\widetilde{\mathcal{E}}\left(r \rho^{\prime}(r)+n \rho(r)+\widetilde{r} \rho^{\prime}(\widetilde{r})+n \rho(\widetilde{r})\right)-\varepsilon \frac{\rho^{\prime}(r)}{r}((x-a) \cdot \nabla u)^{2}\right. \\
-\varepsilon \frac{\rho^{\prime}(\widetilde{r})}{\widetilde{r}}\left(\mathcal{J}_{x}(\widetilde{x}-a) \cdot \nabla u\right)^{2}-\varepsilon|\nabla u|^{2}(\rho(r)+\rho(\widetilde{r})) \\
+\rho(\widetilde{r})\left(Q_{1} \widetilde{\mathcal{E}}-Q_{2}^{i j} \varepsilon u_{x_{i}} u_{x_{j}}\right)=0,
\end{gathered}
$$

where $Q_{1}=\left(\tau_{i k}-\nu_{i k}\right)_{x_{i}}(\widetilde{x}-a)_{k}$ and $Q_{2}^{i j}=\left(\tau_{i k}-\nu_{i k}\right)_{x_{j}}(\widetilde{x}-a)_{k}$, and by rearranging the terms,

$$
\begin{gathered}
-(n-1) \int_{\Omega} \widetilde{\mathcal{E}}(\rho(r)+\rho(\widetilde{r}))+\int_{\Omega} \widetilde{\mathcal{E}}\left(r \rho^{\prime}(r)+\widetilde{r} \rho^{\prime}(\widetilde{r})\right) \\
=\int_{\Omega}\left(\frac{\varepsilon|\nabla u|^{2}}{2}-\frac{\widetilde{W}}{\varepsilon}\right)(\rho(r)+\rho(\widetilde{r})) \\
+\int_{\Omega} \varepsilon\left(\frac{\rho^{\prime}(r)}{r}((x-a) \cdot \nabla u)^{2}+\frac{\rho^{\prime}(\widetilde{r})}{\widetilde{r}}\left(\mathcal{J}_{x}(\widetilde{x}-a) \cdot \nabla u\right)^{2}\right) \\
-\int_{\Omega} \rho(\widetilde{r})\left(Q_{1} \widetilde{\mathcal{E}}-\varepsilon Q_{2}^{i j} u_{x_{i}} u_{x_{j}}\right) .
\end{gathered}
$$

Let $\phi \in C^{1}(\mathbb{R})$ be such that $\phi=1$ for $x \leq \frac{1}{2}, \phi=0$ for $x \geq 1, \phi^{\prime} \leq 0$ on $\mathbb{R}$, and set $\rho(r)=\phi(r / t)$. Using $r \rho^{\prime}(r)=-t(\partial / \partial t) \phi(r / t)$, we then obtain

$$
(n-1) I(t)-t I^{\prime}(t)=-J_{8}(t)-t J_{2}^{\prime}(t)-J_{3}(t),
$$


where

$$
\begin{aligned}
& I(t)=\int_{\Omega} \widetilde{\mathcal{E}}(\phi(r / t)+\phi(\widetilde{r} / t)), \\
& J_{1}(t)=\int_{\Omega}\left(\frac{\widetilde{W}}{\varepsilon}-\frac{\varepsilon|\nabla u|^{2}}{2}\right)(\phi(r / t)+\phi(\widetilde{r} / t)), \\
& J_{2}(t)=\int_{\Omega} \varepsilon\left\{\phi(r / t)\left(\frac{x-a}{r} \cdot \nabla u\right)^{2}+\phi(\widetilde{r} / t)\left(\frac{\mathcal{J}_{x}(\widetilde{x}-a)}{\widetilde{r}} \cdot \nabla u\right)^{2}\right\}, \\
& J_{3}(t)=\int_{\Omega} \phi(\widetilde{r} / t)\left(Q_{1} \widetilde{\mathcal{E}}-Q_{2}^{i j} \varepsilon u_{x_{i}} u_{x_{j}}\right) .
\end{aligned}
$$

Multiplying by $t^{-n}$,

$$
\frac{d}{d t}\left(\frac{1}{t^{n-1}} I(t)\right)=\left(J_{1}+t J_{2}^{\prime}+J_{3}\right) t^{-n} .
$$

Approximate $\phi$ to $\chi_{(-\infty, 1]}$, the characteristic function of $(-\infty, 1]$, to obtain

$$
\begin{gathered}
\frac{d}{d t}\left\{\frac{1}{t^{n-1}}\left(\int_{B_{t}(a)} \tilde{\mathcal{E}}+\int_{\widetilde{B}_{t}(a)} \tilde{\mathcal{E}}\right)\right\} \\
=\frac{1}{t^{n}}\left(\int_{B_{t}(a)}\left(\frac{W}{\varepsilon}-\frac{\varepsilon|\nabla u|^{2}}{2}\right)+\int_{\widetilde{B}_{t}(a)}\left(\frac{\widetilde{W}}{\varepsilon}-\frac{\varepsilon|\nabla u|^{2}}{2}\right)\right) \\
+\frac{1}{t^{n-1}} \frac{d}{d t}\left\{\int_{B_{t}(a)} \varepsilon\left(\frac{x-a}{r} \cdot \nabla u\right)^{2}+\int_{\widetilde{B}_{t}(a)}\left(\frac{\mathcal{J}_{x}(\widetilde{x}-a)}{\widetilde{r}} \cdot \nabla u\right)^{2}\right\} \\
+\frac{1}{t^{n}} \int_{\widetilde{B}_{t}(a)}\left(Q_{1} \widetilde{\mathcal{E}}-\varepsilon Q_{2}^{i j} u_{x_{i}} u_{x_{j}}\right) .
\end{gathered}
$$

The first and second terms in the right-hand side are non-negative by Proposition 1 , hence we obtain

$$
\frac{d}{d t}\left\{\frac{1}{t^{n-1}}\left(\int_{B_{t}(a)} \widetilde{\mathcal{E}}+\int_{\widetilde{B}_{t}(a)} \widetilde{\mathcal{E}}\right)\right\} \geq \frac{1}{t^{n}} \int_{\widetilde{B}_{t}(a)}\left(Q_{1} \widetilde{\mathcal{E}}-\varepsilon Q_{2}^{i j} u_{x_{i}} u_{x_{j}}\right) .
$$

Since $\left|Q_{1}\right|,\left|Q_{2}\right| \leq$ ct on $\widetilde{B}_{t}(a) \subset\left\{\operatorname{dist}(a, \partial \Omega)<2\left(3 \kappa_{\max }\right)^{-1}\right\}$ for a constant $c$ depending only on $\Omega$, one obtains, for a suitably chosen $c_{3}$, that

$$
\frac{d}{d t}\left\{\frac{e^{c_{3} t}}{t^{n-1}}\left(\int_{B_{t}(a)} \widetilde{\mathcal{E}}+\int_{\widetilde{B}_{t}(a)} \tilde{\mathcal{E}}\right)\right\} \geq 0
$$

We next give a proof of Theorem 1 .

Proof. Suppose $x \in \operatorname{spt} \mu$. Let $\delta_{0}>0$ be such that $W(u)<\delta_{0}$ implies $W^{\prime \prime}(u) \geq \kappa / 2$. We claim that, on passing a subsequence, there exist $x_{i} \in \bar{\Omega}$ such that $W\left(u^{\varepsilon_{i}}\left(x_{i}\right)\right) \geq \delta_{0}$ and $\lim _{i \rightarrow \infty} x_{i}=x$. If this were not true, then there would exist some $r>0$ such that $B_{r}(x) \cap\{x \in$ $\left.\bar{\Omega} \mid W\left(u^{\varepsilon_{i}}(x)\right) \geq \delta_{0}\right\}=\emptyset$ for all large $i$, i.e., $W^{\prime \prime}\left(u^{\varepsilon_{i}}(y)\right) \geq \frac{\kappa}{2}$ for $y \in$ $B_{r}(x)$. Let $\phi \in C_{c}^{1}\left(\mathbb{R}^{n} ; \mathbb{R}\right)$ be such that $\phi=1$ on $B_{r / 2}(x), \phi=0$ on 
$\mathbb{R}^{n} \backslash B_{r}(x)$. Differentiate $(2.1)$ with respect to $x_{k}$ and multiply $u_{x_{k}}^{\varepsilon_{i}} \phi^{2}$. After summing over $k$, we have

$$
\int \phi^{2} \varepsilon_{i} u_{x_{k}}^{\varepsilon_{i}} \Delta u_{x_{k}}^{\varepsilon_{i}}=\int \frac{W^{\prime \prime}\left(u^{\varepsilon_{i}}\right)}{\varepsilon_{i}}\left|\nabla u^{\varepsilon_{i}}\right|^{2} \phi^{2} .
$$

Use $W^{\prime \prime} \geq \kappa / 2$ on $B_{r}(x)$ and integrate by parts to obtain

$$
\begin{aligned}
-\varepsilon_{i} \int\left(2 \phi \phi_{x_{l}} u_{x_{l} x_{k}}^{\varepsilon_{i}} u_{x_{k}}^{\varepsilon_{i}}\right. & \left.+\left|\nabla^{2} u^{\varepsilon_{i}}\right|^{2} \phi^{2}\right)+\int_{\partial \Omega} \varepsilon_{i} \phi^{2} \frac{\partial}{\partial \nu}\left(\frac{\left|\nabla u^{\varepsilon_{i}}\right|^{2}}{2}\right) \\
& \geq \frac{\kappa}{2 \varepsilon_{i}} \int\left|\nabla u^{\varepsilon_{i}}\right|^{2} \phi^{2}
\end{aligned}
$$

We use Lemma 1 to conclude that $\frac{\partial}{\partial \nu}\left(\left|\nabla u^{\varepsilon_{i}}\right|^{2}\right) \leq 0$. By the CauchySchwarz inequality, we have

$$
\varepsilon_{i} \int\left|\nabla^{2} u^{\varepsilon_{i}}\right|^{2} \phi^{2}+\frac{\kappa}{2 \varepsilon_{i}} \int\left|\nabla u^{\varepsilon_{i}}\right|^{2} \phi^{2} \leq \varepsilon_{i} c(n) \int|\nabla \phi|^{2}\left|\nabla u^{\varepsilon_{i}}\right|^{2} .
$$

Since the right-hand side is bounded, we have

$$
\int_{B_{r / 2}(x)} \varepsilon_{i}\left|\nabla u^{\varepsilon_{i}}\right|^{2} \leq \varepsilon_{i}^{2} c(n, r) E_{0} \rightarrow 0
$$

as $i \rightarrow \infty$. Repeating the argument, we may conclude that, for any $k>0$ and $s<r / 2$,

$$
\int_{B_{s}(x)}\left|\nabla^{2} u^{\varepsilon_{i}}\right|^{2} \leq c(s, k, r) \varepsilon_{i}^{k}
$$

Thus $\int_{B_{s}(x)} \frac{\left|\tilde{W}^{\prime}\left(u^{\varepsilon_{i}}\right)\right|}{\varepsilon_{i}} \leq c \varepsilon_{i}^{k}$ by the equation (2.1). Since $u^{\varepsilon_{i}}$ is continuous, there is some $a_{j}$ such that $a_{j}-\delta<u^{\varepsilon_{i}}<a_{j}+\delta$ on $B_{r}(x)$. Let $\tilde{a}_{j}$ be the zero of $\widetilde{W}^{\prime}$ close to $a_{j}$. It is easy to check that $\left|\widetilde{a}_{j}-a_{j}\right| \leq c(W) \varepsilon_{i}$. Since $W\left(u^{\varepsilon_{i}}\right) \leq c(W)\left(u^{\varepsilon_{i}}-a_{j}\right)^{2} \leq c(W)\left(\left(u^{\varepsilon_{i}}-\widetilde{a}_{j}\right)^{2}+2\left(u^{\varepsilon_{i}}-\widetilde{a}_{j}\right) a_{j}+\left(\widetilde{a}_{j}-a_{j}\right)^{2}\right)$ and $\left|u^{\varepsilon_{i}}-\widetilde{a}_{j}\right| \leq c(W)\left|\widetilde{W}^{\prime}\left(u^{\varepsilon_{i}}\right)\right|$, one conclude

$\int_{B_{s}(x)} \frac{W\left(u^{\varepsilon_{i}}\right)}{\varepsilon_{i}} \leq c(W) \int\left(\left|u^{\varepsilon_{i}}-\widetilde{a}_{j}\right|+\left|\widetilde{a}_{j}-a_{j}\right|^{2}\right) / \varepsilon_{i} \leq c(W)\left(\varepsilon_{i}^{k}+\varepsilon_{i}\right) \rightarrow 0$ as $i \rightarrow \infty$. Hence we proved that there exists $r>0$ such that

$$
\lim _{i \rightarrow \infty} \int_{B_{r}(x)}\left(\frac{\varepsilon_{i}\left|\nabla u^{\varepsilon_{i}}\right|^{2}}{2}+\frac{W\left(u^{\varepsilon_{i}}\right)}{\varepsilon_{i}}\right)=0 .
$$

This contradicts $x \in \operatorname{spt} \mu$.

We now established that there exists $x_{i} \rightarrow x$ with $W\left(u^{\varepsilon_{i}}\left(x_{i}\right)\right) \geq \delta_{0}>$ 0 . By rescaling $x-x_{i}$ by $1 / \varepsilon_{i}$, we have $\Delta u^{\varepsilon_{i}}=\widetilde{W}^{\prime}\left(u^{\varepsilon_{i}}\right)$ on $B_{1}\left(x_{i}\right) \cap \frac{1}{\varepsilon_{i}} \Omega$. By the standard elliptic estimate, we have $\left\|u^{\varepsilon_{i}}\right\|_{C^{1}\left(B_{1}\right)}$ bounded, and there exists some $c(W)>0$ such that $\int_{B_{1}\left(x_{i}\right)} W\left(u^{\varepsilon_{i}}\right) \geq c(W)$. Rescaling back, this implies $\frac{1}{\varepsilon_{i}^{n-1}} \int_{B_{\varepsilon_{i}}\left(x_{i}\right)} \frac{W\left(u^{\varepsilon_{i}}\right)}{\varepsilon_{i}} \geq c(W)$. Either using the interior 
or boundary monotonicity formula, we obtain, for $x \in \operatorname{spt} \mu$ and $0<$ $r<r_{0}\left(\Omega, c_{1}, c_{2}\right)$,

$$
\frac{c(W)}{2} \leq \frac{1}{r^{n-1}} \int_{B_{r}\left(x_{i}\right)}\left(\frac{\varepsilon_{i}\left|\nabla u^{\varepsilon_{i}}\right|^{2}}{2}+\frac{W\left(u^{\varepsilon_{i}}\right)}{\varepsilon_{i}}\right)
$$

for all sufficiently large $i$, and since $x_{i} \rightarrow x$, the same inequality holds for $\mu\left(B_{r}(x)\right)$ as well. This show the lower bound estimate. The upper estimate follows immediately from the monotonicity formula.

We now prove Theorem 2 and 3.

Proof. We need to prove two statements. For $\alpha>0$, let $(\operatorname{spt} \mu)_{\alpha}$ be the $\alpha$-neighborhood of spt $\mu$ in $\mathbb{R}^{n}$. Suppose that there is some subsequence (denoted by $\left.\left\{x_{j}\right\}_{j=1}^{\infty} \subset \bar{\Omega}\right)$ such that $x_{j} \in \bar{\Omega} \backslash(\operatorname{spt} \mu)_{\alpha}$ and $W\left(u^{\varepsilon_{j}}\left(x_{j}\right)\right) \geq$ $\delta$ for all $j$. By the monotonicity formula and the argument in Theorem 1 , this implies $\mu\left(B_{\frac{\alpha}{2}}(x)\right)>0$, where $x=\lim _{j \rightarrow \infty} x_{j}$. This contradicts $x \in \bar{\Omega} \backslash(\operatorname{spt} \mu)_{\alpha}$. This shows that $\left\{x \in \bar{\Omega} \mid W\left(u^{\varepsilon_{i}}(x)\right) \geq \delta\right\} \subset(\operatorname{spt} \mu)_{\alpha}$ for all large $i$.

Let $\left\{W\left(u^{\varepsilon_{i}}\right) \geq \delta\right\}_{\alpha}$ be the $\alpha$-neighborhood of $\left\{x \in \bar{\Omega} \mid W\left(u^{\varepsilon_{i}}(x)\right) \geq\right.$ $\delta\}$. Assume that there exists some $x \in \operatorname{spt} \mu$ such that $x \notin\left\{W\left(u^{\varepsilon_{i}}\right) \geq\right.$ $\delta\}_{\alpha}$ for some subsequence (denoted by $\left\{u^{\varepsilon_{i}}\right\}_{i=1}^{\infty}$ ). This implies that $W\left(u^{\varepsilon_{i}}(x)\right)<\delta$ on $B_{\frac{\alpha}{2}}(x)$, but the previous argument for Theorem 1 shows that $\mu\left(B_{\frac{\alpha}{4}}(x)\right)=0$ if this is the case. Thus this again contradicts $x \in \operatorname{spt} \mu$. Thus we proved that $\left\{W\left(u^{\varepsilon_{i}}\right) \geq \delta\right\}$ converges spt $\mu$ in the Hausdorff distance. Theorem 3 follows immediately from Theorem 2 and the present proof.

Before giving proof for Theorem 4, let us recall Sternberg \& Zumbrun's result:

Theorem 7. ([11]) Suppose $\left\{u^{\varepsilon_{i}}\right\}_{i=1}^{\infty}$ are critical stable points and assume the conditions in Section 2. Then, there exists $k_{0}$ such that, if we define for $k \geq k_{0}$

$$
A_{i}=\left\{x \in \bar{\Omega} \mid a_{1}^{\varepsilon}+\varepsilon^{k}<u^{\varepsilon_{i}}(x)<a_{2}^{\varepsilon}-\varepsilon^{k}\right\},
$$

$A_{i}$ must be connected. Here, $a_{1}^{\varepsilon}$ and $a_{2}^{\varepsilon}$ are zeros of $\widetilde{W}^{\prime}\left(=W^{\prime}-\lambda_{i} \varepsilon_{i}\right)$.

Due to the lack of strong convergence, it was not clear if the limit interface is connected in [11].

Proof. Assume that spt $\mu$ can be written as two disjoint closed sets $A_{1}$ and $A_{2}$, spt $\mu=A_{1} \cup A_{2}$ and $A_{1} \cap A_{2}=\emptyset$. Then, there exists some $\alpha>0$ such that $\left(A_{1}\right)_{\alpha} \cap\left(A_{2}\right)_{\alpha}=\emptyset$ as well. On $C=\bar{\Omega} \backslash\left(\left(A_{1}\right)_{\alpha} \cup\left(A_{2}\right)_{\alpha}\right)$, for all sufficiently large $i$, we can prove that $\int_{C} \frac{\left|\tilde{W}^{\prime}\left(u^{\varepsilon_{i}}\right)\right|}{\varepsilon_{i}} \leq c \varepsilon_{i}^{k}$ just as in the proof of Theorem 1. This combined with the standard elliptic 
estimates applied to the $\varepsilon$-scale shows that $\left|u^{\varepsilon_{i}}-a_{1}^{\varepsilon}\right|$ or $\left|u^{\varepsilon_{i}}-a_{2}^{\varepsilon}\right|$ on $C$ is bounded by $c \varepsilon^{k}$ for any $k$ with a suitable choice of $c$. Thus, for all large $i, A_{i}$ must be contained both in $\left(A_{1}\right)_{\alpha}$ and $\left(A_{2}\right)_{\alpha}$ and is disjoint with $C$, which means that $A_{i}$ must be disconnected. This contradicts Theorem 7 , thus spt $\mu$ must be connected.

\section{Remarks on $\operatorname{spt} \mu$}

With the assumptions (A1-4), and for double-well potential case (i.e. $N=2$ ), precise properties of $\mu$ are discussed in [5]. We point out a perhaps confusing difference to the reader about [5] and the present article. In [5], we assumed $W^{\prime}$ has only three zeros but no assumption on the shape of domain, and obtained various interior results for the limit interface. In the present article, we assumed $\Omega$ is convex but do not assume $W^{\prime}$ has three zeros, and we obtain a monotonicity formula up to the boundary. In fact, except at the part we obtain the monotonicity formula in [5], the assumption that $W^{\prime}$ has only three zeros is not used in the latter part of [5]. Thus, all the interior results in [5] applies equally under the assumptions of the present article with $N=2$. We summarize the results from [5]. We refer to [9] for the definitions of rectifiable sets and varifolds:

Theorem 8. Suppose (A1-4) are satisfied with $N=2$. Then,

1. $\operatorname{spt} \mu$ is $(n-1)$-rectifiable set.

2. Let $\theta(x)=\lim _{r \rightarrow 0} \frac{1}{\omega_{n-1 r^{n-1}}} \mu\left(B_{r}(x)\right)$ for $x \in \operatorname{spt} \mu$. Here, $\omega_{n-1}$ is the volume of the unit $(n-1)$-dimensional ball in $\mathbb{R}^{n-1}$. Such limit exists at all points $x \in \operatorname{spt} \mu \cap \Omega$, and the value $\theta(x)$ is integer multiple of $\sigma=\int_{a_{1}}^{a_{2}} \sqrt{W(s) / 2} d s$ for $\mathcal{H}^{n-1}$ a.e. on $\Omega \cap \operatorname{spt} \mu$. Moreover, $\theta(x)$ is upper semicontinuous in $\Omega$.

3. Let $u^{0}=\lim _{i \rightarrow \infty} u^{\varepsilon_{i}}$. Then,

$$
\sigma^{-1} \theta(x)= \begin{cases}\text { odd } & \mathcal{H}^{n-1} \text { a.e. } x \in \Omega \cap \partial\left\{u^{0}=a_{1}\right\} \\ \text { even } & \mathcal{H}^{n-1} \text { a.e. } x \in \Omega \cap \operatorname{spt} \mu \backslash \partial\left\{u^{0}=a_{1}\right\}\end{cases}
$$

4. If we define the varifold $V$ with the underlining $(n-1)$-rectifiable set being spt $\mu$ and the weight being $\theta(x)$, the generalized mean curvature $H$ of $V$ in $\Omega$ is given by

$H(x)= \begin{cases}\frac{\lambda_{\infty}}{\theta(x)} \nu^{\infty}(x) & \mathcal{H}^{n-1} \text { a.e. } x \in \Omega \cap \partial\left\{u^{0}=a_{1}\right\}, \\ 0 & \mathcal{H}^{n-1} \text { a.e. } x \in \Omega \cap \operatorname{spt} \mu \backslash \partial\left\{u^{0}=a_{1}\right\} .\end{cases}$

Here, $\lambda_{\infty}=\lim _{i \rightarrow \infty} \lambda_{i}$ and $\nu^{\infty}(x)$ is the unit normal to $\Omega \cap \partial\left\{u^{0}=\right.$ $\left.a_{1}\right\}$. 
It is of interest to point out that the boundary measures

$$
\left(\frac{\varepsilon_{i}\left|\nabla u^{\varepsilon_{i}}\right|^{2}}{2}+\frac{W\left(u^{\varepsilon_{i}}\right)}{\varepsilon_{i}}\right) d \mathcal{H}^{n-1}\left\lfloor_{\partial \Omega}\right.
$$

is uniformly bonded under the assumption (A1-4). This is seen as follows: multiply $(2.1)$ by $\nabla u^{\varepsilon_{i}} \cdot x$. After integration by parts, we have

$$
\int_{\partial \Omega}\left(\frac{\varepsilon_{i}\left|\nabla u^{\varepsilon_{i}}\right|^{2}}{2}+\frac{W\left(u^{\varepsilon_{i}}\right)}{\varepsilon_{i}}\right)=(n-2) \int_{\Omega} \frac{\varepsilon_{i}\left|\nabla u^{\varepsilon_{i}}\right|^{2}}{2}+\int_{\Omega} \lambda_{i}\left(x \cdot \nabla u^{\varepsilon_{i}}\right) .
$$

The right-hand side is uniformly bounded under the assumption (A14). Thus, on passing a subsequence, we have a Radon measure $\mu_{B}$ on $\partial \Omega$ as a limit. Via an argument similar to that of Theorem 1, one may prove

$$
\operatorname{spt} \mu_{B} \subset \operatorname{spt} \mu \cap \partial \Omega .
$$

On the other hand, the precise density properties of $\mu_{B}$ could not be obtained.

\section{REFERENCES}

[1] Cahn, J.W., Hilliard, J.E. Free energy of a nonuniform system I. Interfacial free energy, J. Chem. Phys. 28 (1958) 258267

[2] Casten, R., Holland, C. Instability results for reaction-diffusion equations with Neumann boundary conditions, J. Diff. Eqns. 27 (1978) 266-273

[3] Grüter, M., Jost, J. Allard type regularity results for varifolds with free boundaries, Ann. Scuola Norm. Sup. Pisa Cl. Sci. (4) 13 (1986), no. 1, 129-169

[4] Gilbarg, D., Trudinger, N.S. Elliptic partial differential equations of second order, 2nd Edition, Springer-Verlag (1983)

[5] Hutchinson, J.E., Tonegawa, Y. Convergence of phase interfaces in the van der Waals - Cahn - Hilliard theory, Calc. Var. 10 (2000) 49-84

[6] Modica, L. A gradient bound and a Liouville theorem for nonlinear Poisson equations, Comm. Pure Appl. Math. 38 (1985), no. 5, 679-684

[7] Modica, L. The gradient theory of phase transitions and the minimal interface criterion, Arch. Rational Mech. Anal. 98 (1987), no. 2, 123 142

[8] Modica, L. Monotonicity of the energy for entire solutions of semilinear elliptic equations, Partial differential equations and the calculus of variations, Vol. II, 843-850, Progr. Nonlinear Differential Equations Appl., 2, Birkhauser Boston, (1989)

[9] Simon, L. Lectures on geometric measure theory, Proc. Centre Math. Anal. Australian National Univ. Vol. 3, (1983)

[10] Sternberg, P. The effect of a singular perturbation on nonconvex variational problems, Arch. Rational Mech. Anal. 101 (1988), no. 3, 209-260

[11] Sternberg, P., Zumbrun, K. Connectivity of phase boundaries in strictly convex domains, Arch. Rational Mech. Anal. 141 (1998), no. 4, 375-400

Department of Mathematics

Hokkaido University 
Sapporo 060-0810

Japan

Email: tonegawa@math.sci.hokudai.ac.jp 